Volume 30 (2021) 217-230

DOI: $10.24330 /$ ieja.969907

\title{
ABELIAN GROUPS WITH LEFT COMORPHIC ENDOMORPHISM RINGS
}

\author{
Grigore Călugăreanu and Andrey Chekhlov \\ Received: 24 July 2020; Revised: 21 September 2020; Accepted: 21 September 2020 \\ Communicated by A. Çiğdem Özcan

\begin{abstract}
A ring $R$ is called left comorphic if for every $a \in R$ there exists $b \in R$ such that the left and right annihilators satisfy $R a=l(b)$ and $r(a)=b R$. In this paper, the Abelian groups with left comorphic endomorphism rings are completely determined.
\end{abstract}

Mathematics Subject Classification (2020): 20K30, 20K10, 20K15, 16U99

Keywords: Left comorphic ring, Abelian group, endomorphism ring

\section{Introduction}

An element $a$ of a ring $R$ is called left comorphic (see [1]) if there exists $b \in R$ such that $R a=l(b)$ and $r(a)=b R$. In this case $b$ is called a companion of $a$. Elements satisfying only $r(a)=b R$, were termed right $G$-morphic (see [19]). A ring is left comorphic if so are all its elements. Such rings are studied in [1].

Note that the equality $a b=0$ in any ring $R$ is equivalent to either $R a \subseteq l(b)$ or $b R \subseteq r(a)$.

Symmetrically, an element $a \in R$ is right comorphic if there exists $c \in R$ such that $a R=r(c)$ and $l(a)=R c$. Rings all whose elements are right comorphic are termed right comorphic. A ring is called comorphic if it is both left and right comorphic.

(Von Neumann) regular rings and $\mathbb{Z} /(n)$ for any $n \geq 2$ are comorphic but $\mathbb{Z}$ is not (left or right) comorphic. However, comorphic rings might not be regular: $\mathbb{Z} /(4)$ is comorphic but not regular. Units and idempotents are left (and right) comorphic. More general, if $a^{n}=a$ for some integer $n>1$, then $b=1-a^{n-1}$ is a companion for $a$. The only (co)morphic elements in any (not necessarily commutative) domain are the units and 0 .

It is easy to see that a (commutative) domain is comorphic if and only if it is a division ring, and, a direct product $R=\prod_{i} R_{i}$ is left comorphic if and only if each $R_{i}$ is left comorphic. 
In the sequel, some more definitions will occur. A ring $R$ is called right principally injective (right $P$-injective for short) if, for each $a \in R$ every map $a R \longrightarrow R_{R}$ extends to $R$, or, equivalently, if $\operatorname{lr}(a)=R a$ for each $a \in R$, or, equivalently, if $R a$ is a left annihilator for each $a \in R$. Therefore, $a$ is left comorphic if and only if it is right $P$-injective and right $G$-morphic.

Recall that an element $a \in R$ is called left morphic if $R a=l(b)$ and $l(a)=R b$ for some $b \in R$, term which is also used for rings all whose elements are left morphic. Symmetrically, one defines right morphic elements or rings.

It is clear from the definitions that a commutative ring is comorphic if and only if it is morphic.

Since unit-regular rings are left (and right) morphic, any regular ring which is not unit-regular is left (and right) comorphic, but not left nor right morphic. Such an example is $R=\operatorname{End}_{D}(V)$ where $D$ is a division ring and ${ }_{D} V$ is a vector space of countable dimension.

In [1], an Example 24, which comes back to Björk, of ring which is not left nor right comorphic is given.

A module ${ }_{R} M$ is called image-projective if whenever $\gamma(M) \subseteq \alpha(M)$ for $\alpha, \gamma \in$ $E=\operatorname{End}_{R}(M)$ then $\gamma \in \alpha E$, that is, if an endomorphism $\delta$ exists in the following diagram, when endomorphisms $\alpha$ and $\gamma$ are given.

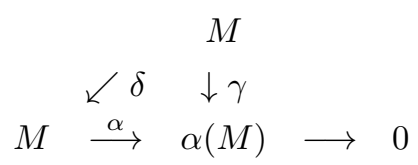

This property was introduced in [14], in order to compare morphic modules to modules with left morphic endomorphism rings. In the same context, a module $M$ with endomorphism ring $E$ is kernel-direct if $\operatorname{ker}(\alpha)$ is a direct summand of $M$, for every $\alpha \in E$. Such modules were recently also called Rickart modules.

From [14] we note the following chart: for any module $M$

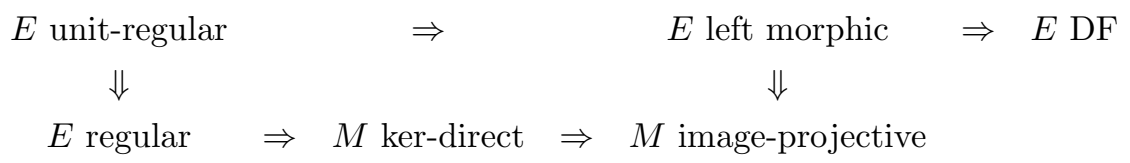

where DF stands for Dedekind finite.

if for every submodule $N$ and every

Finally recall that a module $M$ is quasi-projective if for every submodule $N$ and every $R$-morphism $\gamma: M \rightarrow M / N$, there exists an endomorphism $\delta$ making 
commutative the diagram

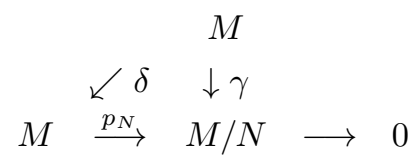

where $p_{N}$ denotes the canonical projection. Clearly quasi-projective modules are image-projective.

In Section 2, a survey of known results is given and (in Theorem 2.10 and Theorem 2.13) all Abelian groups with left comorphic endomorphism rings are determined. In Section 3, we list and comment on three open questions related to this subject.

All groups we consider are Abelian. For the Abelian group definitions and results we refer to [7] and [8]. All the rings we consider are unital. For any subset $X$ of a ring $R, l(X)$ and $r(X)$ denote the left and right annihilators, respectively. For reader's convenience we denote by $\mathbb{Z}(n)$ the cyclic group with $n$ elements and by $\mathbb{Z} /(n)$ the corresponding ring. For an endomorphism $f$ of a group $G,\left.f\right|_{H}$ denotes the restriction homomorphism to a subgroup $H$ of $G$. A direct sum of cyclic groups of the same order is called homocyclic.

\section{The results}

\section{Some immediate examples.}

Since $\operatorname{End}(\mathbb{Z}(n)) \cong \mathbb{Z} /(n)$, the finite cyclic groups have (co)morphic endomorphism rings. Since $\operatorname{End}(\mathbb{Z}) \cong \mathbb{Z}$, the infinite cyclic groups have not left nor right (co)morphic endomorphism rings. Since $\operatorname{End}\left(\mathbb{Z}\left(p^{\infty}\right)\right) \cong \operatorname{End}\left(J_{p}\right) \cong \mathbb{Q}_{p}^{*}$, the commutative domain of $p$-adic integers, which is not a field, the cocyclic divisible group $\mathbb{Z}\left(p^{\infty}\right)$ and the additive group of $p$-adic integers $J_{p}$, do not have left nor right (co)morphic endomorphism rings. Since $\operatorname{End}(\mathbb{Q}) \cong \mathbb{Q}$, is a field, $\mathbb{Q}$ has $($ co)morphic endomorphism ring.

Moreover, since (Von Neumann) regular rings are left (and right) comorphic, the groups which have regular endomorphism ring are also examples of groups with left (and right) comorphic endomorphism rings. These groups are characterized (see $[8], \mathbf{1 1 2 . 7})$ in the following.

Theorem 2.1. (a) $f \in \operatorname{End}(G)$ is regular if and only if both $\operatorname{Im}(\mathrm{f})$ and $\operatorname{ker}(f)$ are direct summands.

(b) If $G$ is not reduced, $\operatorname{End}(G)$ is regular if and only if $G$ is a direct sum of a torsion-free divisible group and an elementary group.

(c) If $G$ is torsion, $\operatorname{End}(G)$ is regular if and only if $G$ is elementary. 
(d) If $G$ is reduced and $\operatorname{End}(G)$ is regular, then $T(G)$ is elementary, $G / T(G)$ is divisible and $\bigoplus_{p} G_{p} \leq G \leq \prod_{p} G_{p}$.

Note that (a) was known to Azumaya as early as 1948.

Recall from [15]

Lemma 2.2. The following conditions are equivalent for a ring $R$.

(i) $R_{R}$ is right $P$-injective.

(ii) $l(r(a))=R a$ for every $a \in R$.

(iii) $r(b) \subseteq r(a), a, b \in R$ implies $R a \subseteq R b$.

We saw above that every left comorphic ring is right P-injective. Then

Proposition 2.3. Let $M_{R}$ be an $R$-module and let $E:=\operatorname{End}_{R}(M)$. If $E$ is left comorphic, then $M$ is image-projective.

Proof. Suppose $M \gamma \subseteq M \alpha$. Then $r_{E}(\alpha) \subseteq r_{E}(\gamma)$ and $E \gamma \subseteq E \alpha$, by the previous lemma.

Therefore, $E$ regular $\Longrightarrow E$ left comorphic $\Longrightarrow M$ image-projective.

Moreover, we recall from [3]:

Proposition 2.4. If $G$ is an image-projective group, then

(1) the divisible part of $G$ is torsion-free;

(2) the torsion part of $G$ is quasi-projective;

(3) if $p$ is a prime such that the p-component $G_{p}$ of $G$ is nonzero, then $G / T(G)$ is $p$-divisible.

Thus, for some classes of image-projective groups, we obtain the following characterizations.

Corollary 2.5. (1) A divisible group is image-projective if and only if it is torsion-free, i.e. a direct sum of copies of $\mathbb{Q}$.

(2) A torsion group is image-projective if and only if it is quasi-projective.

Clearly, all these apply for Abelian groups with left comorphic endomorphism rings. Comparison with Theorem 2.1 gives at once the following.

Corollary 2.6. A divisible group has left (and right) comorphic endomorphism ring if and only if it is torsion-free, i.e. a direct sum of copies of $\mathbb{Q}$. 
This also shows that a reduced torsion group has left comorphic endomorphism ring only if it has homocyclic primary components. However, this way we cannot decide whether these components must be elementary or not.

The structure theorems which follow will show that only the homocyclic condition is also necessary.

We just mention (see [3]): The endomorphism ring of a torsion group $G$ is left morphic if and only if every p-component of $G$ is a homocyclic of finite rank.

In order to state and prove the structure theorems for groups with left comorphic endomorphism ring we first recall some more definitions and known results.

A ring $R$ is called right self-injective if $R_{R}$ is an injective right $R$-module. Since every finitely generated left ideal of a right self-injective ring is an annihilator, any right self-injective ring is right P-injective (this also follows from Lemma 2.2).

We recall from [5] the following:

Lemma 2.7. Suppose $G=A \oplus B \oplus C$ is a group, $f \in \operatorname{Hom}(A, B)$ and $g \in E=$ $\operatorname{End}(G)$, such that $\left.g\right|_{A}=f,\left.g\right|_{B \oplus C}=0$. Then

(1) if $f$ is an epimorphism and $r(l(g)=g E$, then ker $f$ is a direct summand of A;

(2) if $f$ is a monomorphism and $l(r(g))=E g$, then $\operatorname{im} f$ is a direct summand of $B$.

Next we prove:

Proposition 2.8. Let $G=D(G) \oplus R$, where $D(G) \neq 0$ is the divisible part of the group $G$. If the ring $E=\operatorname{End}(G)$ is right P-injective, then $D(G)$ is torsion-free and $R$ is torsion.

Proof. Assume $G=A \oplus B$, where $A \cong \mathbb{Z}\left(p^{\infty}\right)$, and let $\pi: G \rightarrow A$ be the projection. Then $\left.(p \pi)\right|_{A}$ is surjective and so $l(p \pi)=l(\pi)$ and $r(l(p \pi))=r(l(\pi))$. Hence $\pi E=(p \pi) E$ and $\pi=p \pi \gamma$ for some $\gamma \in E$. Then for $0 \neq a \in A[p]$ we have $a=\pi(a)=(p \pi \gamma)(a)=0$, a contradiction. So $D(G)$ is torsion-free.

Next we show that not only $D(G)$ is fully invariant in $G$ but $R$ is also fully invariant in $G$.

We can represent

$$
E=\left\{\left[\begin{array}{cc}
\varphi & \psi \\
0 & \eta
\end{array}\right]: \varphi \in \operatorname{End}(D(G)), \psi \in \operatorname{Hom}(R, D(G)), \eta \in \operatorname{End}(R)\right\} .
$$

By the way of contradiction, take $0 \neq \alpha \in \operatorname{Hom}(R, D(G))$, and for the projection $\pi: G \rightarrow D(G)$, take $\theta=1-\pi$ and $\bar{\alpha}=\pi \alpha \theta=\left[\begin{array}{cc}0 & \alpha \\ 0 & 0\end{array}\right]$. Then $\bar{\alpha} E=$ 
$\left\{\left[\begin{array}{cc}0 & \alpha \eta \\ 0 & 0\end{array}\right]\right\}$ and $l(\bar{\alpha})=\left\{\left(\begin{array}{cc}\varphi^{\prime} & \psi^{\prime} \\ 0 & \eta^{\prime}\end{array}\right): \varphi^{\prime} \alpha=0\right\}$. Chose $\alpha$ such that $\operatorname{im} \alpha \leq$ $D$, where $D$ is a direct summand in $D(G)$ of rank 1 . We go into two cases.

1) If $D=D(G)$ then all its non-zero endomorphisms are monomorphisms and so $\varphi^{\prime}=0$ in $l(\bar{\alpha})$. In this case $\left\{\left[\begin{array}{ll}\varphi & \psi \\ 0 & 0\end{array}\right]\right\} \subseteq r(l(\bar{\alpha})) \neq \bar{\alpha} E$, a contradiction.

2) If $D(G)=A \oplus D$, with $A \neq 0$ then $\left\{\left[\begin{array}{ll}\varphi & \psi \\ 0 & 0\end{array}\right]: \operatorname{im} \varphi \leq D, \operatorname{im} \psi \leq D\right\} \subseteq$ $r(l(\bar{\alpha}))$. If $A \neq 0$ there exists $0 \neq \varphi \in \operatorname{End}(D(G))$, so again $r(l(\bar{\alpha})) \neq \bar{\alpha} E$, a contradiction.

Therefore $E=\operatorname{End}(D(G)) \times \operatorname{End}(R)$.

Finally, if $\operatorname{Hom}(R, D(G))=0$ with torsion-free divisible $D(G)$ and reduced $R$, $R$ must be torsion.

Indeed, $\operatorname{Hom}(A, C) \cong \operatorname{Hom}(A / T(A), C)$ holds for every group $A$ and torsion-free group $C$, and for $n=r_{0}(A), \operatorname{Hom}\left(A, \underset{m}{\bigoplus \mathbb{Q}} \cong \prod_{n}\left(\bigoplus_{m} \mathbb{Q}\right)\right.$.

One more result before our characterizations.

Lemma 2.9. Let $G$ be a group with right P-injective endomorphism ring. Then all its nonzero p-components $G_{p}$ are homocyclic. Moreover, if $G=G_{p} \oplus H$, then $p H=H$. In particular, if $G_{p}=0$ then $p G=G$.

Proof. From Lemma 2.7, (2) follows that each $G_{p}$ is a homocyclic or divisible. Indeed, if $G=A \oplus B \oplus C$ and $f: A \rightarrow B$ is a monomorphism, then $\operatorname{im} f$ is a direct summand of $B$ and so if $A$ and $B$ are cocyclic, then $A \cong B$.

Therefore $G=G_{p} \oplus H$ for some $H \leq G$. Let $\pi: G \rightarrow H$ be the projection. Since $H_{p}=0$ we have $\operatorname{ker}(p \pi)=\operatorname{ker} \pi$ and so $r(p \pi)=r(\pi)$. Hence $l(r(p \pi))=l(r(\pi))$ and so $E(p \pi)=E \pi$. Therefore $\pi=f(p \pi)$ for some $f \in E$ and then $H=\pi(G)=$ $p(f \pi(G))$, whence $p H=H$.

Finally, it was already proved in the previous proposition, that the divisible part of such a group is torsion-free.

Using these we can characterize the non-reduced groups with left comorphic endomorphism ring.

Theorem 2.10. Let $G=D \oplus R$, where $D=D(G) \neq 0$. Then $G$ has left comorphic endomorphic ring $E:=\operatorname{End}(G)$ if and only if $D$ is torsion-free and $R$ is torsion with homocyclic p-components. 
Proof. In view of Proposition 2.8, in order to show the conditions are necessary, it only remains to prove that $R$ has homocyclic $p$-components. The condition is necessary owing to the previous lemma.

As for the converse, we have just to discuss the divisible and primary cases.

1) If $D$ is divisible torsion-free, then $\mathrm{E}(D)$ is regular so left comorphic.

2) If $G$ is a homocyclic $p$-group, then $E$ is right self-injective (see [16]), so it is right $P$-injective and so for every $f \in E$, there is an $g \in E$ such that $E f=l(g)$. Note that $\operatorname{im} g=\operatorname{ker} f$. Indeed, if $\operatorname{im} g \varsubsetneqq \operatorname{ker} f$, then $G / \operatorname{im} g$ is isomorphic to some subgroup of $G$, so there exists $\varphi \in E$ with $\operatorname{ker} \varphi=\operatorname{im} g, \varphi g=0$ and $\varphi \notin E f$. It only remains to show that $r(f)=g E$. Let $\varphi \in r(f)$, i.e. $\operatorname{im} \varphi \leq \operatorname{ker} f=\operatorname{im} g$. Since we already saw that a torsion group all whose $p$-components are homocyclic is (quasi-projective and so) image-projective, $\varphi=g \gamma$ for some $\gamma \in E$ and so $\varphi \in g E$, as desired.

Remarks. 1) The groups in the previous theorem are precisely the groups whose endomorphism rings are right self-injective (see [16] or [10]). Therefore, for endomorphism rings of Abelian not reduced groups, the properties right selfinjective and left comorphic are equivalent.

2) Both $D$ and $R$ above are fully invariant direct summands in the splitting group $G$. Since direct summands of splitting groups are splitting, direct summands of completely decomposable (torsion-free) groups are completely decomposable and direct summands of homocyclic groups are homocyclic (every direct summand of a direct sum of indecomposable $p$-groups is again a direct sum of indecomposable groups), it is easy to see that direct summands of nonreduced groups whose endomorphism rings are left comorphic, also have left comorphic endomorphism rings.

As for reduced groups, the idea of proof of the following theorem is taken from [10] (where Theorem 2.1 describes the endomorphism rings with annihilator condition for $n$-generated left ideals; in particular for right $P$-injective endomorphism rings).

In order to simplify the statement of the next result, we recall a general wellknown construction: for groups $A$ and $B$, set $S_{A}(B)=\sum_{\alpha \in \operatorname{Hom}(A, B)} \alpha(A)$, a fully invariant subgroup of $B$ called the $A$-socle of $B$ (or the trace of $A$ in $B$ ).

Proposition 2.11. A reduced group $G$ has right P-injective endomorphism ring if and only if the following conditions hold:

(1) all p-components of $G$ are homocyclic and the factor group $G / T(G)$ is divisible; 
(2) if $H$ is an endomorphic image of the group $G$ then each homomorphism $H \rightarrow G$ extends to an endomorphism of group $G$;

(3) if $H=\operatorname{ker} f$, where $f \in \operatorname{End}(G)$ and $K=S_{G}(H)$, then $H \leq \operatorname{ker} g$ for each $g \in E$ such that $K \leq \operatorname{ker} g$.

Proof. (1) By Lemma 2.9, $G=G_{p} \oplus F$, where if $G_{p} \neq 0$, then $p F=F$. If $G_{p}=0$, then $p 1_{G}$ is a monomorphism and consequently $p 1_{G}$ is invertible. Hence $p G=G$ and so the factor group $G / T(G)$ is divisible.

(2) Let $H=f(G)$ for $f \in E$ and let $g: H \rightarrow G$ be some homomorphism. Then $g f \in E$ and since $g f \in l(r(f))=E f$ (indeed, if $\alpha \in r(f)$, then $\operatorname{im} \alpha \leq \operatorname{ker} f \leq$ $\operatorname{ker}(g f)$, i.e. $(g f) \alpha=0)$ we obtain $g f=h f$ for some $h \in E$. Hence $g=\left.h\right|_{H}$.

If $f=\bar{f} i$ is the mono-epi decomposition of $f$, the situation is described by the following diagram

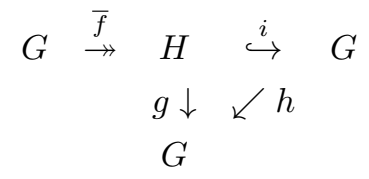

and $g=h i$.

(3) Let $K=S_{G}(H)$ and $K \leq M=\operatorname{ker} g$, where $g \in E$, and let $h \in r(f)$, i.e. $\operatorname{im} h \leq H=\operatorname{ker} f$. Then $\operatorname{im} h \leq K$ and so $g h=0$. Consequently $g \in l(r(f))=E f$, i.e. $g=a f$ for some $a \in E$. But then $H=\operatorname{ker} f \leq \operatorname{ker} g=M$.

Conversely, let $f \in E$ and $g \in l(r(f))$. We have to show that $g \in E f$, i.e. $E f=$ $l(r(f))$. Note that $H=\operatorname{ker} f \leq \operatorname{ker} g$. Indeed, if $K=S_{G}(H)$, then $K \leq \operatorname{ker} g$ since $g \in l(r(f))$, and by hypothesis (3), $H \leq \operatorname{ker} g$. Hence there exists $h \in \operatorname{Hom}(\operatorname{im} f, G)$ such that the following diagram is commutative

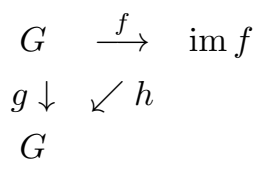

By hypothesis (2), the homomorphism $h$ extends to some endomorphism $q$ of the group $G$. Therefore $g=q f \in E f$.

In order to characterize reduced groups with left comorphic endomorphism rings, we still need a characterization for right G-morphic groups.

Proposition 2.12. A group $G$ has right $G$-morphic endomorphism ring if and only if for every $\alpha \in E=\operatorname{End}(G)$ there exists $\beta \in E$ such that $\operatorname{im} \beta=S_{G}(\operatorname{ker} \alpha)$, and, if $\operatorname{im} \varphi \leq \operatorname{im} \beta$ then $\varphi=\beta \delta$ for some $\delta \in E$. 
Proof. For $\alpha \in E$ suppose there is $\beta \in E$ with $r(\alpha)=\beta E$. Then $\beta(G) \leq S_{G}(\operatorname{ker} \alpha)$ and we show that $K=S_{G}(\operatorname{ker} \alpha) \leq \beta(G)$.

If $x \in K$, then $x=f_{1}\left(g_{1}\right)+\cdots+f_{n}\left(g_{n}\right)$ for some $f_{i} \in \operatorname{Hom}(G, K)$ and $g_{i} \in G$, $i=1, \ldots, n$. Therefore $f_{i} \in r(\alpha)$ and so $f_{i}=\beta \delta_{i}$ for some $\delta_{i} \in E$. Hence $x=\beta \delta_{1}\left(x_{1}\right)+\cdots+\beta \delta_{n}\left(x_{n}\right) \in \beta(G)$ and so $K=\beta(G)$.

Finally, if $\operatorname{im} \varphi \leq \operatorname{im} \beta$ then $\varphi \in r(\alpha)=\beta E$ and so $\varphi=\beta_{\alpha} \delta$ for some $\delta \in E$.

The converse follows from hypothesis and the G-morphic definition.

Combining the previous propositions we finally obtain:

Theorem 2.13. A reduced group $G$ has left comorphic endomorphism ring if and only if the following conditions hold:

(1) all p-components of $G$ are homocyclic and the factor group $G / T(G)$ is divisible;

(2) if $H$ is an endomorphic image of the group $G$ then each homomorphism $H \rightarrow G$ extends to an endomorphism of group $G$;

(3) if $H=\operatorname{ker} f$, where $f \in \operatorname{End}(G)$ and $K=S_{G}(H)$, then $H \leq \operatorname{ker} g$ for each $g \in E$ such that $K \leq \operatorname{ker} g$;

(4) for every $\alpha \in \operatorname{End}(G)$ there exists $\beta \in E$ such that $\operatorname{im} \beta=S_{G}(\operatorname{ker} \alpha)$, and, if $\operatorname{im} \varphi \leq \operatorname{im} \beta$ then $\varphi=\beta \delta$ for some $\delta \in \operatorname{End}(G)$.

Note that we may view a group $G$ which satisfies 1) as a pure subgroup of $F=\prod_{p \in \Pi} G_{p}$, containing $T(F)=\bigoplus_{p \in \Pi} G_{p}$, where $\Pi$ is the set of relevant primes, that is, the condition, known for 50 years, that prevents the characterization of the reduced Abelian groups which have regular endomorphism ring.

From [8]: "A satisfactory, more or less explicit description of reduced groups with regular endomorphism rings seems to be a hard problem. Manifestly, the difficulty lies in singling out the suitable mixed groups between the direct sum and the direct product of their $p$-components".

Remarks. 1) We were not able to prove (or disprove) that the class of reduced groups whose endomorphism rings are left comorphic (characterized in the previous theorem) is closed under direct summands. However, in the last result of this section this is proved for fully invariant direct summands and their complements.

2) Somehow dual to image-projective, we call (see [3]) a module ${ }_{R} M$ imageinjective if $R$-linear maps $\beta(M) \rightarrow M$ extend to $M$ for each $\beta \in E=\operatorname{End}_{R}(M)$. Note that ${ }_{R} R$ is image-injective if and only if $R$ is left P-injective. From the definitions, every right comorphic ring is left P-injective. 
A dual result to Lemma 2.2 holds, quasi-injective groups (i.e. homomorphisms from subgroups can be extended to endomorphisms) were determined by Kil'p [11] and these are either divisible or torsion groups with homocyclic $p$-components. Clearly, quasi-injective groups are image-injective.

It is easy to prove that right $R$-modules with right comorphic endomorphism rings are image-injective. Recall from [3] that if $G$ is an image-injective group, then the torsion part of $G$ is quasi-injective (see [3]). Hence if a torsion group has right comorphic endomorphism ring, its p-components are homocyclic.

Notice that if $E$ is right morphic, then $M_{R}$ is image-injective, and so a p-group has right morphic endomorphism ring if and only if it is homocyclic and finite. The finiteness follows since left (or right) morphic rings are DF. However this fails for left (or right) comorphic rings.

As already mentioned we now prove the following.

Theorem 2.14. If $G=A \oplus B$ is a group with left comorphic endomorphism ring $E$ and $\operatorname{Hom}(B, A)=0$, then $\mathrm{E}(A):=\operatorname{End}(A), \mathrm{E}(B):=\operatorname{End}(B)$ are also left comorphic rings.

Proof. For $\alpha \in \mathrm{E}(A)$ consider $\bar{\alpha} \in E$ with $\left.\bar{\alpha}\right|_{A}=\alpha$ and $\left.\bar{\alpha}\right|_{B}=1_{B}$, i.e., in matrix form, $\bar{\alpha}=\left[\begin{array}{ll}\alpha & 0 \\ 0 & 1\end{array}\right]$. Assume $\beta=\left[\begin{array}{cc}\beta_{1} & 0 \\ \beta_{2} & \beta_{3}\end{array}\right]$ is companion of $\bar{\alpha}$. Since $\bar{\alpha} \beta=\left[\begin{array}{cc}\alpha \beta_{1} & 0 \\ \beta_{2} & \beta_{3}\end{array}\right]$, we have $\beta_{2}=0, \beta_{3}=0$ and $\operatorname{im} \beta_{1} \leq \operatorname{ker} \alpha$. Hereinafter $E \bar{\alpha}=\left\{\left[\begin{array}{cc}\varphi_{1} \alpha & 0 \\ \varphi_{2} \alpha & \varphi_{3}\end{array}\right]\right\}$, where

$$
E=\left\{\left[\begin{array}{cc}
\varphi_{1} & 0 \\
\varphi_{2} & \varphi_{3}
\end{array}\right]: \varphi_{1} \in \mathrm{E}(A), \varphi_{2} \in \operatorname{Hom}(A, B), \varphi_{3} \in \mathrm{E}(B)\right\} .
$$

We show that $\beta_{1}$ is companion of $\alpha$ in $\mathrm{E}(A)$. First, clearly, $\beta_{1} \mathrm{E}(A) \subseteq r(\alpha)$ and $\mathrm{E}(A) \alpha \subseteq l\left(\beta_{1}\right)$. Further, if $f \in r(\alpha)$ then $\left[\begin{array}{ll}f & 0 \\ 0 & 0\end{array}\right] \in r(\bar{\alpha})$, and $\left[\begin{array}{ll}f & 0 \\ 0 & 0\end{array}\right]=$ $\left[\begin{array}{cc}\beta_{1} & 0 \\ 0 & 0\end{array}\right]\left[\begin{array}{cc}\varphi_{1} & 0 \\ \varphi_{2} & \varphi_{3}\end{array}\right]=\left[\begin{array}{cc}\beta_{1} \varphi_{1} & 0 \\ 0 & 0\end{array}\right] \in \beta E$ whence $f=\beta_{1} \varphi_{1} \in \beta_{1} \mathrm{E}(A)$, i.e. $r(\alpha)=\beta_{1} \mathrm{E}(A)$. Finally, if $g \in l\left(\beta_{1}\right)$ then $\left[\begin{array}{ll}g & 0 \\ 0 & 0\end{array}\right] \in l(\beta)$, and $\left[\begin{array}{ll}g & 0 \\ 0 & 0\end{array}\right]=$ $\left[\begin{array}{cc}\varphi_{1} & 0 \\ \varphi_{2} & \varphi_{3}\end{array}\right]\left[\begin{array}{cc}\alpha & 0 \\ 0 & 1\end{array}\right]=\left[\begin{array}{cc}\varphi_{1} \alpha & 0 \\ \varphi_{2} \alpha & \varphi_{3}\end{array}\right] \in E \bar{\alpha}$ whence $g=\varphi_{1} \alpha \in \mathrm{E}(A) \alpha, \varphi_{2} \alpha=0$ $\varphi_{3}=0$. In particular $l\left(\beta_{1}\right)=\mathrm{E}(A) \alpha$. 
The proof for $B$ is somewhat similar.

Let $\delta \in \mathrm{E}(B)$, that is $\bar{\delta}=\left[\begin{array}{ll}1 & 0 \\ 0 & \delta\end{array}\right] \in E$. Assume that $\gamma=\left[\begin{array}{cc}\gamma_{1} & 0 \\ \gamma_{2} & \gamma_{3}\end{array}\right]$ is companion of $\bar{\delta}$. We have $\bar{\delta} \gamma=\left[\begin{array}{cc}\gamma_{1} & 0 \\ \delta \gamma_{2} & \delta \gamma_{3}\end{array}\right]$, so $\gamma_{1}=0$ and $\operatorname{im} \gamma_{2} \leq \operatorname{ker} \delta$, $\operatorname{im} \gamma_{3} \leq \operatorname{ker} \delta$. Hereinafter $E \bar{\delta}=\left\{\left[\begin{array}{cc}\varphi_{1} & 0 \\ \varphi_{2} & \varphi_{3} \delta\end{array}\right]\right\}$. We show that $\gamma_{3}$ is a companion of $\delta$ in $\mathrm{E}(B)$. First, clearly $\gamma_{3} \mathrm{E}(B) \subseteq r(\delta)$ and $\mathrm{E}(B) \delta \subseteq l\left(\gamma_{3}\right)$. Further, if $h \in r(\delta)$ then $\left[\begin{array}{ll}0 & 0 \\ 0 & h\end{array}\right] \in r(\bar{\delta})$, and $\left[\begin{array}{ll}0 & 0 \\ 0 & h\end{array}\right]=\left[\begin{array}{cc}0 & 0 \\ \gamma_{2} & \gamma_{3}\end{array}\right]\left[\begin{array}{cc}\varphi_{1} & 0 \\ \varphi_{2} & \varphi_{3}\end{array}\right]=$ $\left[\begin{array}{cc}0 & 0 \\ \gamma_{2} \varphi_{1}+\gamma_{3} \varphi_{2} & \gamma_{3} \varphi_{3}\end{array}\right] \in \gamma E$ whence $\gamma_{2} \varphi_{1}+\gamma_{3} \varphi_{2}=0, h=\gamma_{3} \varphi_{3} \in \gamma_{3} \mathrm{E}(B)$, i.e. $r(\delta)=\gamma_{3} \mathrm{E}(B)$. Finally if $q \in l\left(\gamma_{3}\right)$ then $\left[\begin{array}{ll}0 & 0 \\ 0 & q\end{array}\right] \in l(\gamma)$, and $\left[\begin{array}{ll}0 & 0 \\ 0 & q\end{array}\right]=$ $\left[\begin{array}{cc}\varphi_{1} & 0 \\ \varphi_{2} & \varphi_{3}\end{array}\right]\left[\begin{array}{ll}1 & 0 \\ 0 & \delta\end{array}\right]=\left[\begin{array}{cc}\varphi_{1} & 0 \\ \varphi_{2} & \varphi_{3} \delta\end{array}\right] \in E \bar{\delta}$ whence $q=\varphi_{3} \delta \in \mathrm{E}(B) \delta$.

Observe that a similar statement holds for modules. The proof given above holds verbatim with the obvious changes.

In closing, related to Proposition 2.3, it is worth mentioning (with a straightforward proof)

Proposition 2.15. Direct summands of image-projective modules are image-projective.

\section{Open questions}

A. If the torsion-free rank of a mixed group is finite, a progress (for groups between the direct sum and the direct product of their $p$-components) was made in [9] for the characterization of S-local groups whose endomorphism rings are right PP (principal projective). A definite answer to this question was obtained in terms of a maximal independent torsion-free subset of $G$ and an associated matrix algebra.

Can we characterize in a similar way, the S-local (mixed) groups whose endomorphism rings are left (or right) comorphic?

B. In [13], it is proved that for an idempotent $e \in R$, an element $a \in e R e$ is left morphic in $e R e$ if and only if $a+\bar{e}$ is left morphic in $R$, and, as a consequence, corners of left morphic rings are also left morphic. 
If for $a \in e R e, a+\bar{e}$ is left morphic in $R$, there is an element $b \in R$ such that $l(a+\bar{e})=R b$ and $l(b)=R(a+\bar{e})$. The key ingredient in the proof of the sufficiency is that we can show $b \in e R e$.

However, in the left comorphic case, we can only prove $b \in e R$, not $b \in e R e$, i.e. the companion of an element in a corner, might not belong to this corner. Hence, it is unlikely that corners of left comorphic rings are left comorphic.

We were not able to prove (or disprove) whether the class of Abelian groups with left comorphic endomorphism ring is closed (or not) under direct summands. In searching for a counterexample, according to our results in the previous section, one has to take a reduced group and a direct summand which is not fully invariant. C. There are many classes of rings, e.g. regular, left (or right) morphic, Baer, Rickart, whose definitions were (the last 50 years or so) transferred to modules (see [12], [14], [17], [18]). Some of these were further transferred to Abelian categories (e.g. [4] and [6]).

Morphic modules were defined asking each endomorphism to satisfy the dual of the Noether isomorphism theorem, i.e. $M / \operatorname{im}(f) \cong \operatorname{ker} f$ for every $f \in \operatorname{End}_{R}(M)$, and, a ring was called left (or right) morphic if ${ }_{R} R$ (respectively $R_{R}$ ) is morphic. This way, morphic modules and $R$-modules whose endomorphism ring is left (or right) morphic are different notions.

The morphic Abelian groups and the Abelian groups with left morphic endomorphism ring were studied in [2] and [3]. It turned out that for large classes of Abelian groups these notions coincide.

When it comes to left (or right) comorphic rings, the easy way to transfer this to modules is to consider modules whose endomorphism ring is left (or right) comorphic, and this was done for Abelian groups in the previous section.

A natural question remains: can we define left (or right) comorphic modules in such a way that restricting this to the modules ${ }_{R} R$ (respectively $R_{R}$ ) we recover the above definition for rings?

Acknowledgement. The authors would like to thank the referee for the valuable suggestions and comments.

\section{References}

[1] M. Alkan, W. K. Nicholson, and A. Ç. Özcan, Comorphic rings, J. Algebra Appl., 17(4) (2018), 1850075 (21 pp).

[2] G. Călugăreanu, Morphic abelian groups, J. Algebra Appl., 9(2) (2010), 185193. 
[3] G. Călugăreanu, Abelian groups with left morphic endomorphism ring, J. Algebra Appl., 17(9) (2018), 1850176 (8 pp).

[4] G. Călugăreanu and L. Pop, Morphic objects in categories, Bull. Math. Soc. Sci. Math. Roumanie, 56(104)(2) (2013), 173-180.

[5] A. R. Chekhlov, Abelian groups with annihilator ideals of endomorphism rings, Sib. Math. J., 59(2) (2018), 363-367.

[6] S. Dăscălescu, C. Năstăsescu, A. Tudorache and L. Dăuş, Relative regular objects in categories, Appl. Categ. Structures, 14(5-6) (2006), 567-577.

[7] L. Fuchs, Infinite Abelian Groups. Vol. I., Academic Press, New York-London, 1970.

[8] L. Fuchs, Infinite Abelian Groups. Vol. II., Academic Press, New York-London, 1973.

[9] S. Glaz and W. Wickless, Regular and principal projective endomorphism rings of mixed abelian groups, Comm. Algebra, 22(4) (1994), 1161-1176.

[10] A.V. Ivanov, Abelian groups with self-injective endomorphism rings and endomorphism rings with annihilator condition, In: Abelian Groups and Modules [Russian], Tomsk. Gos. Univ., Tomsk, (1982), 93-109.

[11] M.A. Kil'p, Quasi-injective abelian groups [Russian], Vestnik Moskov. Univ. Ser. I Mat. Meh., 22(3) (1967), 3-4.

[12] G. Lee, S. T. Rizvi and C. S. Roman, Rickart modules, Comm. Algebra, 38(11) (2010), 4005-4027.

[13] W. K. Nicholson and E. Sánchez Campos, Rings with the dual of the isomorphism theorem, J. Algebra, 271(1) (2004), 391-406.

[14] W. K. Nicholson, A survey of morphic modules and rings, Advances in Ring Theory (Nanjing 2004), World Sci. Publ., Hackensack, NJ, (2005), 167-180.

[15] W. K. Nicholson and M. F. Yousif, Principally injective rings, J. Algebra, 174 (1995), 77-93.

[16] K. M. Rangaswamy, Abelian groups with self-injective endomorphism rings, Lect. Notes. Math., 372 (1974), 595-604.

[17] S. T. Rizvi and C. S. Roman, Baer and quasi-Baer modules, Comm. Algebra, 32(1) (2004), 103-123.

[18] J. Zelmanowitz, Regular modules, Trans. Amer. Math. Soc., 163 (1972), 341355.

[19] H. Zhu and N. Ding, Generalized morphic rings and their applications, Comm. Algebra, 35(9) (2007), 2820-2837. 
Grigore Călugăreanu (Corresponding Author)

Department of Mathematics

Faculty of Mathematics and Computer Science

Babeş-Bolyai University

40084 Cluj-Napoca, Romania

e-mail: calu@math.ubbcluj.ro

\section{Andrey Chekhlov}

Department of Mathematics

Faculty of Mechanics and Mathematics

Tomsk State University

Tomsk, Russia

e-mail: cheklov@math.tsu.ru 